\title{
Widely Linear Cooperative Spectrum Sensing for Cognitive Radio Networks
}

\author{
Angela Sara Cacciapuoti*, Marcello Caleffi* and Luigi Paura*† \\ *Department of Biomedical, Electronics and Telecommunications Engineering \\ University of Naples Federico II, Naples, Italy \\ ${ }^{\dagger}$ Laboratorio Nazionale di Comunicazioni Multimediali (CNIT), Naples, Italy \\ Email:\{angelasara.cacciapuoti, marcello.caleffi, paura\}@unina.it
}

\begin{abstract}
The Spectrum Sensing is a fundamental functionality of the Cognitive Radio (CR) paradigm since a reliable detection of the presence of spectral holes is a mandatory requirement to avoid harmful interferences to licensed users. In this paper, we address this problem by proposing a widely linear (WL) cooperative strategy at a fusion center side where the CR user statistics have been received by means of multipath fading channels. The proposed strategy jointly elaborates the received signal and its conjugate version to take advantage by the information contained in the statistical pseudo-covariance function of the received signal. We design the WL strategy to maximize a modified deflection coefficient and we compare it with a strictly linear (SL) cooperative strategy. Results of numerical simulations show that the WL fusion-rule outperforms the SL one in operative conditions of practical interest.
\end{abstract}

\section{INTRODUCTION}

In the last years, the Cognitive Radio (CR) paradigm [1], [2] has attracted a huge attention since it appears to be a viable and effective solution to assure an efficient use of the radio spectrum. The $\mathrm{CR}$ paradigm proposes to enhance the spectrum utilization efficiency by discovering spectrum holes (i.e. licensed bands not used at a particular place and/or time) and by exploiting them for the unlicensed (CR) user communications. It is worth to highlight that the CR users can use these spectral holes if they do not cause any significant interference to the transmissions of the licensed users (primary users). Moreover, when the primary users become active the $\mathrm{CR}$ users have to vacate the frequency bands. Therefore, the spectrum sensing is a fundamental functionality of CR paradigm and it is a challenging issue due to the wireless propagations effects.

Recently, several spectrum sensing strategies [2]-[5] based on the cooperative approach have been proposed to counteract the wireless propagations effects by exploiting the spatial diversity among the CR users. In centralized cooperative techniques, every CR user performs its own local spectrum sensing measurements independently and sends them to a fusion center. The fusion center combines the sensing information collected from the CR users to perform the decision on the presence of the primary user. In [3]-[5] the energy detection has been adopted as local spectrum sensing measurement since it does

This work is partially supported by the Italian National Project "Global e Reliable End to End e-Commerce e On Line Service Platform (GRECO)" and by the project "Finanziamento per l'Avvio di Ricerche Originali (FARO)". not require any a-priori knowledge about the primary user signal and exhibits a low complexity.

[3] proposes two detection schemes: a linear soft combination and an interesting softened hard one, assuming an ideal transmission between each CR user and the fusion center.

In [4], [5], the authors propose two interesting fusion detection schemes, which optimize the detection performance by means of a linear (L) combination of the energies measured by CR users, assuming a fading flat-flat channel model between each CR user and the fusion center. In the first scheme, the combining weights are designed to maximize the detection probability for a fixed false-alarm probability. In the second technique, instead, the combining weights are designed to optimize a modified deflection coefficient The authors show that the two strategies perform almost the same, even if the second one exhibits a lower complexity.

In this paper, we generalize the model considered in [4], [5] by adopting the more reasonable multipath channel model between each $\mathrm{CR}$ user and the fusion center to take into account the bandwidth scarcity of the management channel for the cognitive procedure. The adopted model induces us to propose as combining fusion rule the widely-linear (WL) processing to take advantage by the non-circular properties associated with the local spectrum sensing statistics. In fact, when the information signal and/or the disturbance are improper the $\mathrm{L}$ receivers can be outperformed by the WL ones that, by jointly elaborating the received signal and its conjugate version, exploit also the information contained in the statistical pseudo-covariance function [6]-[8]. According to the criterion adopted for the L processing in [4], [5], we design the WL combining weights to maximize at the fusion center side a deflection coefficient modified to account for the cognitive radio context. Moreover, we develop the $\mathrm{L}$ cooperative decision rule proposed in [4], [5], referred to as strictly linear (SL) rule, for the adopted multipath channel model for the sake of completeness. Finally, basing on Monte Carlo simulations, we compare the WL decision strategy with the SL one, showing that the WL detector outperforms the SL one with a small complexity increase assuring so, in operative conditions of practical interest, less interference to the primary user.

The rest of the paper is organized as follows: in section II we describe the system model, whereas in section III we develop 
the proposed WL cooperative technique. In section IV we develop the analysis of the SL cooperative rule and in section $\mathrm{V}$ we validate our analysis by comparing the two strategies. Finally conclusions and future works are drawn in section VI.

\section{SYSTEM MODEL}

Let us consider a Cognitive Radio Network with $M$ CR users.

\section{A. CR User Model}

The base-band discrete received signal $x_{i}(k)$ at the $i$ th secondary user can be written as in [4], [5]:

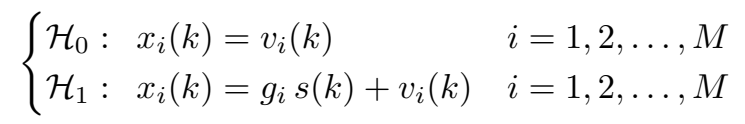

where $s(k)$ is the the primary user's signal, $g_{i}$ is the complex flat-flat channel coefficient and $v_{i}(k)$ is the complex zero-mean additive white Gaussian noise, $v_{i}(k) \sim \mathcal{C N}\left(0, \sigma_{i}^{2}\right)$, assumed circular (or proper), i.e. $E\left[v_{i}(n) v_{i}(m)\right]=0, \forall n, m \in Z$.

Each secondary user adopts an energy detector to reveal the presence of the primary user, namely $u_{i}(q)=$ $\sum_{k=q N_{s}}^{q N_{s}+N-1}\left|x_{i}(k)\right|^{2}$ where the number $N$ of samples in the detection interval is the maximum integer not greater than $\tau f_{s}$ [9], with $\tau$ the available sensing time and $f_{s}$ the sampling frequency and $N_{s}>N$ indicates the number of samples after which a new spectrum sensing process starts. Accounting for the Central Limit Theorem (CLT), for large $N, u_{i}(q)$ can be approximated by a Gaussian random variable (r.v.) with mean and variance ${ }^{1}$ given respectively by:

$$
\begin{gathered}
E\left[u_{i}(q) \mid \mathcal{H}_{j}\right]= \begin{cases}N \sigma_{i}^{2} & \mathcal{H}_{0} \\
\left|g_{i}\right|^{2} E_{s}+N \sigma_{i}^{2} & \mathcal{H}_{1}\end{cases} \\
\operatorname{Var}\left[u_{i}(q) \mid \mathcal{H}_{j}\right]= \begin{cases}N \sigma_{i}^{4} & \mathcal{H}_{0} \\
2\left|g_{i}\right|^{2} E_{s} \sigma_{i}^{2}+N \sigma_{i}^{4} & \mathcal{H}_{1}\end{cases}
\end{gathered}
$$

Remark: although $s(k)$ is assumed deterministic and unknown as in [4], [5], if $s(k)$ is modeled as a sequence of independent and identically distributed (i.i.d) r.v., $\left\{u_{i}(q)\right\}$ can be still approximated by Gaussian r.v. [9] and therefore the subsequent analysis will continue to hold.

\section{B. Fusion Model}

The statistics $\left\{u_{i}(q)\right\}$ are transmitted by the CR users to the fusion center through multipath fading channels. We assume according to [4], [5] that the transmissions of the different CR users are orthogonal each other. Note that this assumption has been considered only for the sake of simplicity since the proposed analysis can be easily extended. The base-band signal at the RF front-end of the fusion center received from $i$ th $\mathrm{CR}$ user can be written as:

$$
y_{i}(l)=\sum_{n=0}^{L_{g_{i}}-1} u_{i}(l-n) h_{i}(n)+n_{i}(l), \quad i \in\{1, \ldots, M\}
$$

\footnotetext{
${ }^{1} E\left[\left|v_{i}(k)\right|^{4}\right]=E\left[\left(\operatorname{Re}\left(v_{i}(k)\right)^{2}+\operatorname{Im}\left(v_{i}(k)\right)^{2}\right)^{2}\right]=2 \sigma_{i}^{4}$, taking into account the notable result valid for zero-mean real Gaussian random variable.
}

where $l$-th is the arbitrary sampling instant, $n_{i}(l)$ is the circular, complex zero-mean, spatially uncorrelated, additive white Gaussian noise, $n_{i}(l) \sim \mathcal{C N}\left(0, \delta_{i}^{2}\right)$, and $\left\{h_{i}(n)\right\}$ is the discrete-time, finite multipath channel impulse response with length $L_{g_{i}}$.

Without loss of generality, we assume that $u_{i}(l)$ is a i.i.d. random sequence, that $u_{i}\left(l_{1}\right)$ and $u_{j}\left(l_{2}\right)$ are spatially uncorrelated, and that the transmitted statistic $\left\{u_{i}(l)\right\}$ are independent from the additive noise $\left\{n_{i}(l)\right\}$ [3]-[5]. In the following, to simplify the notation, we indicate with $L_{g}$ the maximum value among $L_{g_{i}}$. Since via CLT $\left\{u_{i}(l)\right\}$ are normal r.v., $y_{i}(l)$ is a complex Gaussian r.v.. At the fusion center, due to the multipath channel model, we jointly elaborate $L_{e}$ consecutive received samples for each CR user namely $\mathbf{y}_{i}(l) \triangleq\left[y_{i}(l), y_{i}(l-1), \ldots y_{i}\left(l-L_{e}+1\right)\right]^{T} \in \mathcal{C}^{L_{e}}$, which, from (4), can be expressed as:

$$
\mathbf{y}_{i}(l)=\mathbf{H}_{i} \mathbf{u}_{i}(l)+\mathbf{n}_{i}(l), \quad i \in\{1, \ldots, M\}
$$

where

$$
\mathbf{H}_{i} \triangleq\left[\begin{array}{ccccc}
h_{i}(0) & \ldots & h_{i}\left(L_{g}-1\right) & 0 & 0 \\
0 & h_{i}(0) & \ldots & h_{i}\left(L_{g}-1\right) & 0 \\
\vdots & \ddots & \ldots & \ddots & \vdots \\
0 & 0 & h_{i}(0) & \ldots & h_{i}\left(L_{g}-1\right)
\end{array}\right]
$$

is a Toeplitz matrix $\in \mathcal{C}^{L_{e} \times\left(L_{e}+L_{g}-1\right)}, \quad \mathbf{u}_{i}(l) \triangleq$ $\left[u_{i}(l), u_{i}(l-1), \ldots, u_{i}\left(l-L_{e}-L_{g}+2\right)\right]^{T} \in \mathcal{R}^{L_{e}+L_{g}-1}$ and, finally, $\mathbf{n}_{i}(l) \triangleq\left[n_{i}(l), n_{i}(l-1), \ldots, n_{i}\left(l-L_{e}+1\right)\right]^{T} \in \mathcal{C}^{L_{e}}$. If we define $\mathbf{H} \triangleq \operatorname{diag}\left[\mathbf{H}_{1} \mathbf{H}_{2} \ldots \mathbf{H}_{M}\right] \quad \in$ $\mathcal{C}^{M L_{e} \times M\left(L_{e}+L_{g}-1\right)}, \mathbf{u}(l) \triangleq\left[\mathbf{u}_{1}^{T}(l) \mathbf{u}_{2}^{T}(l) \ldots \mathbf{u}_{M}^{T}(l)\right]^{T} \in$ $\mathcal{R}^{M\left(L_{e}+L_{g}-1\right)}$, and $\mathbf{n}(l) \triangleq\left[\mathbf{n}_{1}^{T}(l) \mathbf{n}_{2}^{T}(l) \ldots \mathbf{n}_{M}^{T}(l)\right]^{T} \in$ $\mathcal{C}^{M L_{e}}$, we can rewrite (5) in a more compact form:

$$
\mathbf{y}(l)=\mathbf{H} \mathbf{u}(l)+\mathbf{n}(l)
$$

where $\mathbf{y}(l) \triangleq\left[\mathbf{y}_{1}^{T}(l) \mathbf{y}_{2}^{T}(l) \ldots \mathbf{y}_{M}^{T}(l)\right]^{T} \in \mathcal{C}^{M L_{e}}$. Taking into account (2) and (3), the mean and the covariance matrix of the complex Gaussian random vector $\mathbf{y}(l)$, are equal to:

$$
\boldsymbol{\mu}_{\mathcal{H}_{j}}=E\left[\mathbf{y}(l) \mid \mathcal{H}_{j}\right]=\mathbf{H} E\left[\mathbf{u}(l) \mid \mathcal{H}_{j}\right]=\mathbf{H} \boldsymbol{\eta}_{\mathcal{H}_{j}} \in \mathbb{C}^{M L_{e}}
$$

where

$\boldsymbol{\eta}_{\mathcal{H}_{j}}= \begin{cases}{\left[N \sigma_{1}^{2} \mathbf{1}^{T} \ldots N \sigma_{M}^{2} \mathbf{1}^{T}\right]^{T}} & \mathcal{H}_{0} \\ {\left[\left(E_{s}\left|g_{1}\right|^{2}+N \sigma_{1}^{2}\right) \mathbf{1}^{T} \ldots\left(E_{s}\left|g_{M}\right|^{2}+N \sigma_{M}^{2}\right) \mathbf{1}^{T}\right]^{T}} & \mathcal{H}_{1}\end{cases}$

with $\mathbf{1} \triangleq[11 \ldots 1]^{T} \in \mathcal{R}^{L_{e}+L_{g}-1}$ and

$$
\begin{aligned}
\mathbf{C}_{\mathbf{y} \mid \mathcal{H}_{j}} & \triangleq E\left[(\mathbf{y}(l)-\boldsymbol{\mu})(\mathbf{y}(l)-\boldsymbol{\mu})^{H} \mid \mathcal{H}_{j}\right] \in \mathbb{C}^{M L_{e} \times M L_{e}} \\
& =\mathbf{H ~ C}_{\mathbf{u} \mid \mathcal{H}_{j}} \mathbf{H}^{H}+\mathbf{R}_{\mathbf{n}}
\end{aligned}
$$

where the noise correlation matrix and the signal one are

$$
\mathbf{R}_{\mathbf{n}}=\operatorname{diag}\left[\mathbf{R}_{\mathbf{n}_{1}} \mathbf{R}_{\mathbf{n}_{2}} \ldots \mathbf{R}_{\mathbf{n}_{M}}\right] \in \mathbb{R}^{M L_{e} \times M L_{e}}
$$




$$
\begin{aligned}
\mathbf{C}_{\mathbf{u} \mid \mathcal{H}_{j}} & =\operatorname{diag}\left[\mathbf{C}_{\mathbf{u}_{1} \mid \mathcal{H}_{j}} \ldots \mathbf{C}_{\mathbf{u}_{M} \mid \mathcal{H}_{j}}\right] \\
& \in \mathbb{R}^{M\left(L_{e}+L_{g}-1\right) \times M\left(L_{e}+L_{g}-1\right)}
\end{aligned}
$$

with

$$
\mathbf{R}_{\mathbf{n}_{i}}=\delta_{i}^{2} \mathbf{I}_{\left\{L_{e} \times L_{e}\right\}}
$$

and

$$
\mathbf{C}_{\mathbf{u}_{i} \mid \mathcal{H}_{j}}= \begin{cases}N \sigma_{i}^{4} \mathbf{I}_{\left\{L_{e}+L_{g}-1\right\}} & \mathcal{H}_{0} \\ \left(N \sigma_{i}^{4}+2 E_{s}\left|g_{i}\right|^{2} \sigma_{i}^{2}\right) \mathbf{I}_{\left\{L_{e}+L_{g}-1\right\}} & \mathcal{H}_{1}\end{cases}
$$

Moreover, since $\left\{\mathbf{u}_{i}(l)\right\}$ are real, the pseudo-autocovariance matrix of $\mathbf{y}(l)$ is different from zero and it is given by:

$$
\begin{aligned}
\mathbf{C}_{\mathbf{y}^{*} \mid \mathcal{H}_{j}} & \triangleq E\left[(\mathbf{y}(l)-\boldsymbol{\mu})(\mathbf{y}(l)-\boldsymbol{\mu})^{T} \mid \mathcal{H}_{j}\right] \in \mathbb{C}^{M L_{e} \times M L_{e}} \\
& =\mathbf{H ~ C}_{\mathbf{u}^{*} \mid \mathcal{H}_{j}} \mathbf{H}^{T}+\mathbf{R}_{\mathbf{n}^{*}}=\mathbf{H ~ C}_{\mathbf{u} \mid \mathcal{H}_{j}} \mathbf{H}^{T}
\end{aligned}
$$

where the last equality takes into account that $\mathbf{u}(l)$ is a realvalue vector and that $\mathbf{n}(l)$ is a proper random vector.

\section{Widely Linear Cooperative Spectrum Sensing}

In this section the proposed Widely Linear cooperative strategy is developed by resorting to a modified deflection coefficient.

\section{A. Widely Linear Global Decision}

As mentioned in section I, the WL processing can take advantage (with respect to the SL one) of the correlation between the received signal and its conjugate version, namely of the information contained in their statistical pseudo-covariance function. The WL global test statistic at the fusion center can be expressed as:

$$
y_{\mathrm{wl}}(l)=\mathbf{f}_{1}^{H} \mathbf{y}(l)+\mathbf{f}_{2}^{H} \mathbf{y}^{*}(l)=\mathbf{f}^{H} \mathbf{y}_{a}(l)=2 \operatorname{Re}\left[\mathbf{f}_{1}^{H} \mathbf{y}(l)\right]
$$

where $\mathbf{y}_{a}(l) \triangleq\left[\mathbf{y}^{T}(l) \mathbf{y}^{H}(l)\right]^{T} \in \mathbb{C}^{2 M L_{e}}$ is the augmented received vector and the augmented processing vector $\mathbf{f} \triangleq$ $\left[\begin{array}{ll}\mathbf{f}_{1}^{T} & \mathbf{f}_{2}^{T}\end{array}\right]^{T}$ is equal to $\mathbf{f}=\left[\begin{array}{ll}\mathbf{f}_{1}^{T} & \mathbf{f}_{1}^{H}\end{array}\right]^{T} \in \mathbb{C}^{2 M L_{e}}$ since for real information symbols $\mathbf{f}_{2}=\mathbf{f}_{1}^{*}$ namely $\mathbf{f}$ exhibits the conjugate symmetric property [8]. We note that unlike the SL processing, in this case the statistic (15) is real. In order to characterize the Gaussian r.v. $y_{\mathrm{wl}}(l)$, it is necessary to characterize $\mathbf{y}_{a}(l)$. At this end, basing on (6), $\mathbf{y}_{a}(l)$ can be written as:

$$
\mathbf{y}_{a}(l)=\underbrace{\left[\begin{array}{c}
\mathbf{H} \\
\mathbf{H}^{*}
\end{array}\right]}_{\mathbf{H}_{a}} \mathbf{u}(l)+\underbrace{\left[\begin{array}{c}
\mathbf{n}(l) \\
\mathbf{n}^{*}(l)
\end{array}\right]}_{\boldsymbol{n}_{a}(l)}=\mathbf{H}_{a} \mathbf{u}(l)+\mathbf{n}_{a}(l)
$$

and its mean and its covariance matrix are respectively:

$$
\widetilde{\boldsymbol{\mu}}_{\mathcal{H}_{j}} \triangleq E\left[\mathbf{y}_{a}(l) \mid \mathcal{H}_{j}\right]=\mathbf{H}_{a} E\left[\mathbf{u}(l) \mid \mathcal{H}_{j}\right]=\mathbf{H}_{a} \boldsymbol{\eta}_{\mathcal{H}_{j}} \in \mathbb{C}^{2 M L_{e}}
$$

with $\boldsymbol{\eta}_{\mathcal{H}_{j}}$ given by (8) and

$$
\begin{aligned}
\mathcal{C}_{\mathbf{y}_{a} \mid \mathcal{H}_{j}} & \triangleq E\left[\left(\mathbf{y}_{a}(l)-\widetilde{\boldsymbol{\mu}}\right)\left(\mathbf{y}_{a}(l)-\widetilde{\boldsymbol{\mu}}\right)^{H} \mid \mathcal{H}_{j}\right] \in \mathbb{C}^{2 M L_{e} \times 2 M L_{e}} \\
& =\mathbf{H}_{a} \mathbf{C}_{\mathbf{u} \mid \mathcal{H}_{j}} \mathbf{H}_{a}^{H}+\mathbf{R}_{\mathbf{n}_{a}}
\end{aligned}
$$

where the augmented noise correlation matrix is

$$
\mathbf{R}_{\mathbf{n}_{a}}=\left[\begin{array}{cc}
\mathbf{R}_{\mathbf{n}} & \mathbf{0}_{\{M \times M\}} \\
\mathbf{0}_{\{M \times M\}} & \mathbf{R}_{\mathbf{n}}
\end{array}\right] \in \mathbb{C}^{2 M L_{e} \times 2 M L_{e}}
$$

and $\mathbf{C}_{\mathbf{u} \mid \mathcal{H}_{j}}$ is given in (11).

Accounting of (17), (18) we can characterize $y_{\mathrm{wl}}(l)$ in (15):

$$
\begin{aligned}
& \mu_{\mathrm{w}|| \mathcal{H}_{j}} \triangleq E\left[y_{\mathrm{wl}}(l) \mid \mathcal{H}_{j}\right]=\mathbf{f}^{H} E\left[\mathbf{y}_{a}(l) \mid \mathcal{H}_{j}\right]=\mathbf{f}^{H} \tilde{\boldsymbol{\mu}}_{\mathcal{H}_{j}} \\
& \varphi_{\mathrm{w}|| \mathcal{H}_{j}}^{2} \triangleq \operatorname{Var}\left[y_{\mathrm{wl}}(l) \mid \mathcal{H}_{j}\right]= \\
& \mathbf{f}^{H} E\left[\left(\mathbf{y}_{a}(l)-\widetilde{\boldsymbol{\mu}}\right)\left(\mathbf{y}_{a}(l)-\widetilde{\boldsymbol{\mu}}\right)^{H} \mid \mathcal{H}_{j}\right] \mathbf{f}=\mathbf{f}^{H} \mathbf{C}_{\mathbf{y}_{a} \mid \mathcal{H}_{j}} \mathbf{f}
\end{aligned}
$$

The WL-based decision rule at the fusion center is:

$$
y_{\mathrm{wl}}(l) \gtreqless \mathcal{H}_{1} \gamma_{0} \gamma_{\mathrm{wl}}
$$

where $\gamma_{\mathrm{wl}}$ is the decision threshold. By taking into account (20) and (21), the false-alarm probability $P_{f}$ and the detection one $P_{d}$ can be expressed as:

$$
\begin{aligned}
P_{f} & \triangleq P\left[y_{\mathrm{wl}}(l)>\gamma_{\mathrm{wl}} \mid \mathcal{H}_{0}\right]=Q\left(\frac{\gamma_{\mathrm{wl}}-\mu_{\mathrm{wl} \mid \mathcal{H}_{0}}}{\sqrt{\varphi_{\mathrm{w}|| \mathcal{H}_{0}}^{2}}}\right) \\
& =Q\left(\frac{\gamma_{\mathrm{wl}}-\mathbf{f}^{H} \widetilde{\boldsymbol{\mu}}_{\mathcal{H}_{0}}}{\sqrt{\mathbf{f}^{H} \mathbf{C}_{\mathbf{y}_{a} \mid \mathcal{H}_{0}} \mathbf{f}}}\right) \\
P_{d} & \triangleq P\left[y_{\mathrm{wl}}(l)>\gamma_{\mathrm{wl}} \mid \mathcal{H}_{1}\right]=Q\left(\frac{\gamma_{\mathrm{wl}}-\mu_{\mathrm{w}|| \mathcal{H}_{1}}}{\sqrt{\varphi_{\mathrm{wl} \mid \mathcal{H}_{1}}^{2}}}\right) \\
& =Q\left(\frac{\gamma_{\mathrm{wl}}-\mathbf{f}^{H} \widetilde{\boldsymbol{\mu}}_{\mathcal{H}_{1}}}{\sqrt{\mathbf{f}^{H} \mathbf{C}_{\mathbf{y}_{a} \mid \mathcal{H}_{1}} \mathbf{f}}}\right)
\end{aligned}
$$

In the next subsection we propose to single out the weighting vector $\mathbf{f}$ according to a selected optimization criterion.

\section{B. Widely Linear Optimization}

Since the processing vector $\mathbf{f}$ affects the statistics of the global test statistic $y_{\mathrm{wl}}(l)$ as can be recognized from (20) and (21) and therefore it affects the shape of the Probability Density Function (PDF) of $y_{\mathrm{wl}}(l)$, an effective optimization criterion consists of maximizing the deflection coefficient for a fixed false-alarm probability [10], [11]. In many cases of interest it can be show that such a criterion leads to the optimum likelihood ratio test. As in [4], [5], to increase the detection sensitivity, we adopt the following modified deflection coefficient (MDC):

$$
\begin{aligned}
d_{a}^{2}(\mathbf{f}) & \triangleq \frac{\left\{E\left[y_{\mathrm{wl}}(l) \mid \mathcal{H}_{1}\right]-E\left[y_{\mathrm{wl}}(l) \mid \mathcal{H}_{0}\right]\right\}^{2}}{\operatorname{Var}\left[y_{\mathrm{wl}}(l) \mid \mathcal{H}_{1}\right]}=\frac{\left[\mu_{\mathrm{wl} \mid \mathcal{H}_{1}}-\mu_{\mathrm{wl} \mid \mathcal{H}_{0}}\right]^{2}}{\mathbf{f}^{H} \mathbf{C}_{\mathbf{y}_{a} \mid \mathcal{H}_{1}} \mathbf{f}} \\
& =\frac{\left[\mathbf{f}^{H}\left(\widetilde{\boldsymbol{\mu}}_{\mathcal{H}_{1}}-\widetilde{\boldsymbol{\mu}}_{\mathcal{H}_{0}}\right)\right]^{2}}{\mathbf{f}^{H} \mathbf{C}_{\mathbf{y}_{a} \mid \mathcal{H}_{1}} \mathbf{f}}
\end{aligned}
$$

For accurate inference, we maximize (25) under the unit-norm constraint on the weight vector, namely

$$
\max _{\mathbf{f}} d_{a}^{2}(\mathbf{f}) \quad \text { subject to } \mathbf{f}^{H} \mathbf{f}=1
$$


By using the Cauchy-Schwartz's inequality ${ }^{2}$ one has:

$$
\begin{gathered}
d_{a}^{2}(\mathbf{f})=\frac{\left[\left(\mathbf{C}_{\mathbf{y}_{a} \mid \mathcal{H}_{1}}^{1 / 2} \mathbf{f}\right)^{H} \mathbf{C}_{\mathbf{y}_{a} \mid \mathcal{H}_{1}}^{-1 / 2}\left(\widetilde{\boldsymbol{\mu}}_{\mathcal{H}_{1}}-\widetilde{\boldsymbol{\mu}}_{\mathcal{H}_{0}}\right)\right]^{2}}{\mathbf{f}^{H} \mathbf{C}_{\mathbf{y}_{a} \mid \mathcal{H}_{1}} \mathbf{f}} \\
\leq\left(\widetilde{\boldsymbol{\mu}}_{\mathcal{H}_{1}}-\widetilde{\boldsymbol{\mu}}_{\mathcal{H}_{0}}\right)^{H} \mathbf{C}_{\mathbf{y}_{a} \mid \mathcal{H}_{1}}^{-1}\left(\widetilde{\boldsymbol{\mu}}_{\mathcal{H}_{1}}-\widetilde{\boldsymbol{\mu}}_{\mathcal{H}_{0}}\right)
\end{gathered}
$$

where the equality holds if

$$
\mathbf{f}_{\text {wl-opt }}=\xi \mathbf{C}_{\mathbf{y}_{a} \mid \mathcal{H}_{1}}^{-1}\left(\widetilde{\boldsymbol{\mu}}_{\mathcal{H}_{1}}-\widetilde{\boldsymbol{\mu}}_{\mathcal{H}_{0}}\right)
$$

The constraint $\mathbf{f}^{H} \mathbf{f}=1$ can be satisfied by setting the constant $\xi=1 /\left\|\mathbf{C}_{\mathbf{y}_{a} \mid \mathcal{H}_{1}}^{-1}\left(\widetilde{\boldsymbol{\mu}}_{\mathcal{H}_{1}}-\widetilde{\boldsymbol{\mu}}_{\mathcal{H}_{0}}\right)\right\|_{2}^{2}$. Therefore from (27) the modified deflection coefficient is maximized by

$$
\mathbf{f}_{\mathrm{wl}-\mathrm{opt}}=\frac{\mathbf{C}_{\mathbf{y}_{a} \mid \mathcal{H}_{1}}^{-1}\left(\widetilde{\boldsymbol{\mu}}_{\mathcal{H}_{1}}-\widetilde{\boldsymbol{\mu}}_{\mathcal{H}_{0}}\right)}{\|\left.\mathbf{C}_{\mathbf{y}_{a} \mid \mathcal{H}_{1}}^{-1}\left(\widetilde{\boldsymbol{\mu}}_{\mathcal{H}_{1}}-\widetilde{\boldsymbol{\mu}}_{\mathcal{H}_{0}}\right)\right|_{2} ^{2}}
$$

Once $\mathbf{f}$ is determined, from (23) we can set the decision threshold to obtain a given false-alarm probability:

$$
\gamma_{\mathrm{wl}}=Q^{-1}\left(P_{f}\right) \sqrt{\mathbf{f}_{\mathrm{wl}-\mathrm{opt}}^{H} \mathbf{C}_{\mathbf{y}_{a} \mid \mathcal{H}_{0}} \mathbf{f}_{\mathrm{wl}-\mathrm{opt}}}+\mathbf{f}_{\mathrm{wl}-\mathrm{opt}}^{H} \tilde{\boldsymbol{\mu}}_{\mathcal{H}_{0}}
$$

IV. Strictly Linear Cooperative Spectrum Sensing In this section, the considered channel model is adopted also for the recently proposed SL cooperative decision rule [4], [5], in order to compare its performances with the ones of the WL scheme. A SL fusion rule can be written as

$$
y_{\mathrm{sl}}(l)=\mathbf{w}^{H} \mathbf{y}(l)
$$

where $\mathbf{w}$ is chosen in order to maximize the MDC: where $\gamma$ is the decision threshold and the real-part operator takes into account that the transmitted energy values are real. The false-alarm and the detection probabilities can be expressed as:

$$
P_{f} \triangleq P\left[\operatorname{Re}\left[y_{\mathrm{sl}}(l)\right]>\gamma \mid \mathcal{H}_{0}\right]=Q\left(\frac{\gamma-E\left[\operatorname{Re}\left[y_{\mathrm{sl}}(l)\right] \mid \mathcal{H}_{0}\right]}{\sqrt{\operatorname{Var}\left[\operatorname{Re}\left[y_{\mathrm{sl}}(l)\right] \mid \mathcal{H}_{0}\right]}}\right)
$$

$$
P_{d} \triangleq P\left[\operatorname{Re}\left[y_{\mathrm{sl}}(l)\right]>\gamma \mid \mathcal{H}_{1}\right]=Q\left(\frac{\gamma-E\left[\operatorname{Re}\left[y_{\mathrm{sl}}(l)\right] \mid \mathcal{H}_{1}\right]}{\sqrt{\operatorname{Var}\left[\operatorname{Re}\left[y_{\mathrm{sl}}(l)\right] \mid \mathcal{H}_{1}\right]}}\right)
$$

where the statistical characterization of the r.v. $\operatorname{Re}\left[y_{\mathrm{sl}}(l)\right]$ is:

$$
\mu_{\mathrm{sl} \mid \mathcal{H}_{j}} \triangleq E\left[\operatorname{Re}\left[y_{\mathrm{sl}}(l)\right] \mid \mathcal{H}_{j}\right]=\operatorname{Re}\left(\mathbf{w}_{\text {sl-opt }}^{H} \boldsymbol{\mu}_{\mathcal{H}_{j}}\right)
$$

where $\boldsymbol{\mu}_{\mathcal{H}_{j}}$ is given by (7) and

$$
\begin{aligned}
& \varphi_{\text {sl } \mid \mathcal{H}_{j}}^{2} \triangleq \operatorname{Var}\left[\operatorname{Re}\left[y_{\text {sl }}(l)\right] \mid \mathcal{H}_{j}\right] \\
& =\frac{\mathbf{w}_{\text {sl-opt }}^{H} \mathbf{C}_{\mathbf{y} \mid \mathcal{H}_{j}} \mathbf{w}_{\text {sl-opt }}+\operatorname{Re}\left[\mathbf{w}_{\text {sl-opt }}^{H} \mathbf{C}_{\mathbf{y}^{*} \mid \mathcal{H}_{j}} \mathbf{w}_{\text {sl-opt }}^{*}\right]}{2}
\end{aligned}
$$

where we have used the equality $\operatorname{Re}^{2}(z)=\left(|z|^{2}+\operatorname{Re}\left(z^{2}\right)\right) / 2$ and $\mathbf{C}_{\mathbf{y} \mid \mathcal{H}_{j}}$ and $\mathbf{C}_{\mathbf{y}^{*} \mid \mathcal{H}_{j}}$ are given by (9) and (14), respectively. From (36) we can derive the expression of the threshold $\gamma$ :

$$
\begin{aligned}
& \gamma=Q^{-1}\left(P_{f}\right) \sqrt{\varphi_{\text {sl } \mid \mathcal{H}_{0}}^{2}}+\mu_{\text {sl } \mid \mathcal{H}_{0}}= \\
& \quad \frac{\sqrt{2}}{2} Q^{-1}\left(P_{f}\right) \sqrt{\mathbf{w}_{\text {sl-opt }}^{H} \mathbf{C}_{\mathbf{y} \mid \mathcal{H}_{0}} \mathbf{w}_{\text {sl-opt }}+\operatorname{Re}\left[\mathbf{w}_{\text {sl-opt }}^{H} \mathbf{C}_{\mathbf{y}^{*} \mid \mathcal{H}_{0}} \mathbf{w}_{\text {sl-opt }}^{*}\right]} \\
& \quad+\operatorname{Re}\left(\mathbf{w}_{\text {sl-opt }}^{H} \boldsymbol{\mu}_{\mathcal{H}_{0}}\right)
\end{aligned}
$$

\section{NuMERICAL RESUlts}

$d^{2}(\mathbf{w}) \triangleq \frac{\left|E\left[y_{\mathrm{sl}}(l) \mid \mathcal{H}_{1}\right]-E\left[y_{\mathrm{sl}}(l) \mid \mathcal{H}_{0}\right]\right|^{2}}{\operatorname{Var}\left[y_{\mathrm{sl}}(l) \mid \mathcal{H}_{1}\right]}=\frac{\left|\mathbf{w}^{H}\left(\boldsymbol{\mu}_{\mathcal{H}_{1}}-\boldsymbol{\mu}_{\mathcal{H}_{0}}\right)\right|^{2}}{\mathbf{w}^{H} \mathbf{C}_{\mathbf{y} \mid \mathcal{H}_{1}} \mathbf{w}}$

where $\boldsymbol{\mu}_{\mathcal{H}_{j}}$ and $\mathbf{C}_{\mathbf{y} \mid \mathcal{H}_{j}}$ are given by (7) and (9) respectively. We note that, unlike the WL case, in the SL one $y_{\mathrm{sl}}(l)$ is a complex r.v. and this justifies the presence of the absolute value at the numerator of (32).

For accurate inference, we maximize (32) under the unit-norm constraint on the weight vector, namely

$$
\max _{\mathbf{w}} d^{2}(\mathbf{w}) \quad \text { subject to } \mathbf{w}^{H} \mathbf{w}=1
$$

and by following the same reasoning of section III-B, we have that the MDC is maximized by

$$
\mathbf{w}_{\text {sl-opt }}=\frac{\mathbf{C}_{\mathbf{y} \mid \mathcal{H}_{1}}^{-1}\left(\boldsymbol{\mu}_{\mathcal{H}_{1}}-\boldsymbol{\mu}_{\mathcal{H}_{0}}\right)}{\|\left.\mathbf{C}_{\mathbf{y} \mid \mathcal{H}_{1}}^{-1}\left(\boldsymbol{\mu}_{\mathcal{H}_{1}}-\boldsymbol{\mu}_{\mathcal{H}_{0}}\right)\right|_{2} ^{2}}
$$

The SL test at the fusion center is

$$
\operatorname{Re}\left[y_{\mathrm{sl}}(l)\right] \gtreqless \mathcal{H}_{1} \gamma
$$

${ }^{2}$ Given the vectors $\mathbf{a}, \mathbf{b} \in \mathbb{C}^{n}$, the Cauchy-Schwartz's inequality states that $\left|\mathbf{a}^{H} \mathbf{b}\right|^{2} \leq\|\mathbf{a}\|^{2}\|\mathbf{b}\|^{2}$, where the upper bound is achieved by $\mathbf{b}=\xi \mathbf{a}$, with $\xi \in \mathbb{C}$.
In this section we validate our analysis through Monte Carlo
simulations. In particular, we consider the following detection strategies: the proposed WL technique, the SL one, the optimum test, namely the likelihood ratio test (LRT), and, finally, a non-cooperative strategy (NC) in which each CR user takes an autonomous decision basing only on its local energy measurement. We note, that the NC probabilities are obtained averaging with respect to all the CR users.

We consider a $\mathrm{CR}$ network with $M=4 \mathrm{CR}$ users where each CR user independently senses the targeted spectrum band. Each experiment consists of $10^{6}$ independent Monte Carlo runs and in each run the noise samples are randomly and independently generated according to a zero-mean complex normal distribution. Moreover, in each run the channel between each CR user and the primary user is generated according to a complex normal distribution $\mathcal{C N}(0,1)$ characterized by independent and equally distributed in phase and quadrature components. For simplicity we assume that the transmitted primary signal has unit power $|s(k)|^{2}=1$. The lengths of the FIR filters that model the links between the CR users and the fusion center are set to $L_{g}=2$ and the tap values of the channel vectors are randomly and independently generated in each run according to a zero-mean complex circular 


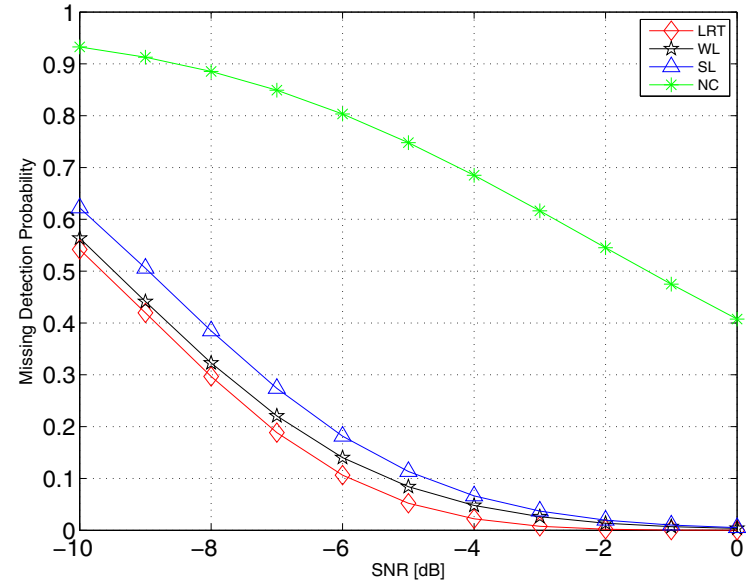

Fig. 1. Missing Detection Probability versus SNR, with false-alarm probability constraint $P_{f}=10^{-2}$

Gaussian process. We set $L_{e}=3$ in order to assure a short processing delay and, at the same time, a reasonable number of consecutive received samples to elaborate. As in [3]-[5], the instantaneous signal-to-noise ratio (SNR) at each CR user is defined as $\mathrm{SNR}_{i}=\left|g_{i}\right|^{2} E s /\left(N \sigma_{i}^{2}\right), \quad i \in\{1, \ldots, M\}$.

Figure 1 shows the probability of missing-detection against the average SNR for a fixed false-alarm probability equal to $P_{f}=10^{-2}$. The results are obtained by considering different noise variances at the fusion center, i.e. $\delta_{1}^{2}=0.9$, $\delta_{2}^{2}=1.3, \delta_{3}^{2}=1$ and $\delta_{4}^{2}=2$, and by setting the number of samples used in the detection interval equal to $N=20$. We underline that different noise variances account for different CR sensing capabilities at the fusion center side, conferring so generality to the analysis. The results show that the NC strategy performs notably worse than the cooperative ones. Moreover, the WL fusion-rule outperforms the SL one in operative conditions of practical interest, namely, low SNR, assuring so less interference to the primary user. Finally, the LRT is confirmed to be the best decision rule at the price of a huge computational complexity.

Figure 2 shows the missing-detection probability against the false-alarm probability. The results are obtained setting $N=$ 50 and assuming that the $\mathrm{CR}$ users are characterized by different values of the average SNR, $\{-10.5,-11,-11.5,-12\}$ $\mathrm{dB}$, in order to exploit different local sensing capabilities that affect the fusion decision. Moreover the noise variances at the fusion center are set equal to one, i.e $\delta_{i}^{2}=\delta^{2}=$ $1 \forall i \in\{1, \ldots, M\}$. The results confirm the suitability of the proposed WL technique, which is able to assure better sensing performances with respect to the SL one also in presence of different sensing capabilities.

\section{CONClusions}

In this paper, we have addressed the Spectrum Sensing problem in CR networks by proposing a widely linear (WL) cooperative strategy at a fusion center side where the CR user statistics have been received by means of multipath fading channels. The WL strategy has been designed to maximize a

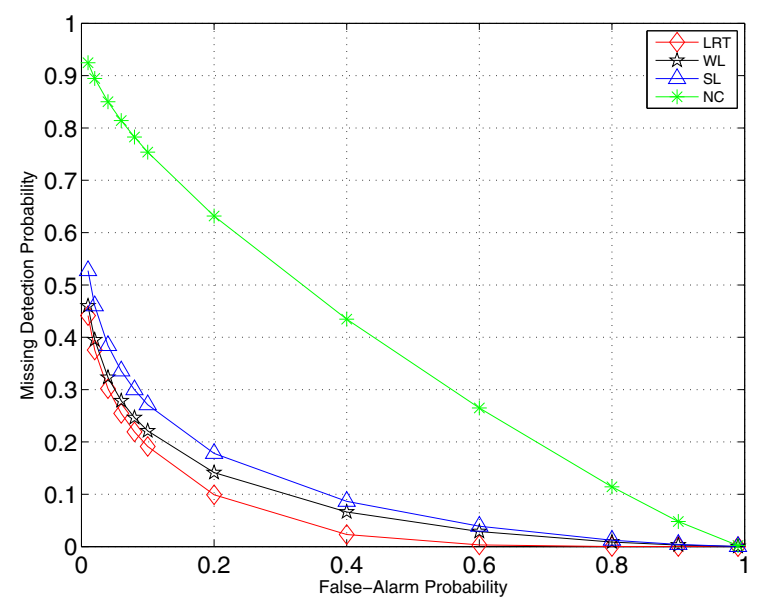

Fig. 2. Missing Detection Probability versus False-Alarm Probability

modified deflection coefficient and it has been compared with a strictly linear (SL) cooperative strategy designed according to the same criterion. Numerical results show that the WL fusion-rule outperforms the SL one in operative conditions of practical interest assuring so less interference to the primary user. Some interesting future works will be to design a fully decentralized scenario and to extend our analysis to a multipath fading channel among the primary user and the CR users, considering potentially joint detection approaches.

\section{REFERENCES}

[1] J. M. III and J. G.Q. Maguire, "Cognitive radio: making software radios more personal," Personal Communications, IEEE, vol. 6, no. 4, pp. 13 -18, Aug 1999.

[2] S. Haykin, "Cognitive radio: brain-empowered wireless communications," IEEE Journal on Selected Areas in Communications, vol. 23, no. 2, pp. $201-220$, Feb. 2005.

[3] J. Man and Y. Li, "Soft combination and detection for cooperative spectrum sensing in cognitive radio natworks," in Global Telecommunications Conference, 2007. GLOBECOM '07. IEEE, Nov. 2007, pp. 3139-3143.

[4] Z. Quan, S. Cui, and A. H. Sayed, "An optimal strategy for cooperative spectrum sensing in cognitive radio networks," in Global Telecommunications Conference, 2007. GLOBECOM '07. IEEE, Nov. 2007, pp. 2947 $-2951$.

[5] - "Optimal linear cooperation for spectrum sensing in cognitive radio networks," Selected Topics in Signal Processing, IEEE Journal of, vol. 2, no. 1, pp. $28-40$, Feb. 2008.

[6] B. Picinbono and P. Chevalier, "Widely linear estimation with complex data," IEEE Trans. Signal Processing, vol. 43, no. 8, pp. 2030-2033, Aug. 1995.

[7] P. J. Schreier, L. L. Scharf, and C. T. Mullis, "Detection and estimation of improper complex random signals," IEEE Trans. Inform. Theory, vol. 51, no. 1, pp. 306-312, Jan. 2005.

[8] A. S. Cacciapuoti, G. Gelli, L. Paura, and F. Verde, "Finite-sample performance analysis of widely-linear multiuser receivers for ds-cdma systems," IEEE Trans. Signal Processing, vol. 56, no. 4, pp. 1572-1588, Apr. 2008.

[9] Y.-C. Liang, Y. Zeng, E. C. Peh, and A. T. Hoang, "Sensing-throughput tradeoff for cognitive radio networks," IEEE Transactions on Wireless Communications, vol. 7, no. 4, pp. 1326 -1337, April 2008.

[10] B. Picinbono, "On deflection as a performance criterion in detection," IEEE Transactions on Aerospace and Electronic Systems, vol. 31, no. 3, pp. $1072-1081$, Jul 1995.

[11] S. M. Kay, Fundamentals of Statistical Signal Processing II: Detection Theory. Englewood Cliffs, NJ: Prentice-Hall, 1998. 